drug use; ii) negative effects in general; iii) problems with drugs and sex. Patients were offered the opportunity to see a drug worker in the clinic, who collected more information about drugs used. Questionnaires completed between July 2014 and August 2015 were analysed.

Results 335 questionnaires were completed, but 59 excluded because of lack of patient identifiers. 170 of 276 (62\%) reported recreational drug use. Of these 170, 38 (22\%) reported negative effects in general, $31(18 \%)$ reported problems with drugs and sex. However, these two groups were not identical and 14 reporting problems with sex answered "no" to the question about general problems. Excluding alcohol, 66 had drug details recorded: 16 had not reported problem use. Drugs associated with Chemsex such as GBL, Mephedrone, Ketamine and Crystal Methamphetamine were frequently identified.

Discussion/conclusions A simple questionnaire can identify problem drug use in a substantial proportion of MSM attending sexual health services. Asking specifically about problems relating to sex as well as general negative effects appears to offer a complementary approach. However, not all MSM who use "chems" will self-identify as having problem use, requiring vigilance on the part of clinicians.

\section{P205 MANAGEMENT OF STI OUTBREAKS. WHAT CAN WE LEARN FROM EACH OTHER? A QUALITATIVE STUDY IN THE UK}

${ }^{1}$ Hannah Loftus*, ${ }^{2}$ Kelly Mackenzie, ${ }^{3}$ lan Simms, ${ }^{4,3}$ Jackie Cassell. 'Sheffield Teaching Hospitals NHS Foundation Trust, Sheffield, UK; ${ }^{2}$ School of Health and Related Research, University of Sheffield, Sheffield, UK; ${ }^{3}$ Public Health England, London, UK; ${ }^{4}$ Division of Primary Care and Public Health, Brighton and Sussex Medical School, Brighton, UK

\subsection{6/sextrans-2016-052718.253}

Background/introduction When outbreaks of sexually transmitted infections (STIs) are identified, effective management in a timely manner is essential for bringing the outbreak under control. Challenges to achieving this may vary, needing different approaches.

Aim(s)/objectives To review the management of outbreaks contrasting by setting and sexual orientation in order to inform responsive guidance.

Methods We interviewed clinicians and public health professionals who had recently been involved in identifying and managing STI outbreaks in the United Kingdom. Transcripts were analysed using thematic analysis.

Results Ten outbreaks were reviewed. The combination of public health teams' wider outbreak expertise and clinic staff's knowledge of the local population was essential when developing management strategies. Partner notification, mainly by health advisers, was very achievable in smaller heterosexual outbreaks but proved challenging in MSM focussed outbreaks where use of mobile apps or anonymous sex was common. Publicity campaigns via social media platforms and third sector organisations were employed although quantifying their impact was difficult. Education of local physicians resulted in syphilis referrals to sexual health services via ophthalmology, gastroenterology, oral and maxillofacial surgery and general practice. Enhanced surveillance enabled venue identification but was time consuming for clinic staff. In gonorrhoea outbreaks, the use of dual NAAT testing as part of the chlamydia screening programme enabled case finding.

Discussion/conclusion Traditional management strategies remain important but as the use of social media increases, novel strategies for managing outbreaks are needed. Education of other professionals is essential to maximise case finding.

\section{P206 'DOCTOR, I THINK I'M ALLERGIC TO PENICILLIN' - PRIMARY SYPHILIS IN THE THIRD TRIMESTER}

Adele Wolujewicz*, Christine Bates. Royal Liverpool University Hospital, Liverpool, UK

\subsection{6/sextrans-2016-052718.254}

Case Report We present a case of primary syphilis in the third trimester of pregnancy requiring penicillin desensitisation. A 34year old woman was contacted by provider referral as a syphilis contact. She was 30 weeks pregnant. Both HIV and syphilis serology were negative at booking. She had presented to a community clinic with a sore vulva one week before and had empirical aciclovir for possible genital herpes. In our clinic, examination showed a small, non-indurated ulcer. Dark-ground microscopy was not done. A syphilis antibody screen was requested and reported positive six days later. On recall, repeat examination showed a larger, indurated ulcer. Treatment for primary syphilis was advised before confirmatory testing. However, the patient reported a possible reaction to penicillin. This was also documented by her general practitioner but the reaction was unknown. The next day she was admitted for penicillin desensitisation and the first dose of benzathine penicillin. Urgent referral to foetal medicine was made. Treponema pallidum was later detected by PCR on a vulval swab. Syphilis serology was reported as RPR 1:8 and TPPA 1:80. HIV serology and HSV PCR were negative. A second dose of benzathine penicillin was administered a week later, followed by 45 minutes of observation. After delivery at term, the neonate received 10 days of benzyl penicillin.

Discussion Learning points: 1. Exclude syphilis in anyone with genital ulceration, particularly in pregnancy. 2. Consult Immunology for advice on desensitisation regimen. 3. Write a syphilis birth plan as recommended by new BASHH guidelines

\section{P207 CHLAMYDIA AND OUR 'BEST FRIENDS FOREVER' POLICY}

Andrew de Burgh-Thomas, Angela Arthur*. Gloucestershire Care Services, Gloucester, UK

10.1136/sextrans-2016-052718.255

Background/introduction Our chlamydia screening target was challenging.

Aim(s)/Objectives To test more under 25 year olds in our area. To assess the number of new positives from the intervention and the potential impact on our target.

Methods We implemented a policy that all $<25$ yo's were offered kits for their friends. We developed kits with a 'site code' BFF (Best Friend Forever) enabling laboratory tracking. We developed a local code to determine if the offer was made and accepted/declined.

Results From Dec to Feb 2016 we saw $3072<25$ yo patients. We recorded that we offered BFF kits to 32\% (989). They were accepted by $28 \%$ (277) who took 415 kits (average 1.5 kits each). Of these $15 \%$ (62) were returned and the chlamydia positivity in these kits was $11.2 \%$ (7). From these 7 positives via contact tracing 5 additional positives were identified. On average there are 0.6 chlamydia positives/case identified. If we extrapolate this we expect 6 additional chlamydia positives. From our intervention 7 cases, 5 chlamydia positive partners and an 
estimated 6 additional chlamydia positive partners would have been identified totalling 18 .

Discussion/Conclusion Only 1/3rd of our target group were offered, we calculate 54 new chlamydia positives (18\% of target) if offered to all. Kits costs $£ 1.30$, we distributed 415 costing $£ 539$. We identified 12 patients $(7+5)$ and estimate an extra 6 would have been found so each chlamydia positive costs $£ 30$ $£ 45$ (test kit only).

\section{P208 AUDIT OF THE MANAGEMENT OF HIV POSITIVE PREGNANT WOMEN IN A LOW PREVALENCE SETTING}

Kasey Redler*, Rebecca Hallgren, Rebecca Swingler.

Gloucestershire Hospitals NHS Foundation Trust, Gloucester, UK.

\subsection{6/sextrans-2016-052718.256}

Background/introduction HIV Maternal to child transmission (MTCT) has reduced from 25\% in 1993 to $0.57 \%$ in 2007 due to increased intervention in pregnancy. Compliance with BHIVA guidelines requires multidisciplinary care, which could be a challenge in areas with low HIV prevalence.

Aim(s)/objectives To audit the management of HIV positive pregnant women in a large district general hospital (DGH) against BHIVA guidelines.

Methods Retrospective audit of all HIV positive women giving birth at this DGH between September 2010 and October 2015.

Results 21 women identified. Diagnosis: 100\% screened for STI, hepatitis C, VZV and HIV. Treatment: $81 \%$ on HAART at start of pregnancy, $100 \%$ of the four women not on HAART were started on treatment during pregnancy. At start of pregnancy $61 \%(\mathrm{n}=13$ ) had a viral load $<50 \mathrm{RNA}$ copies $/ \mathrm{ml}$, by the end of pregnancy this increased to $86 \%(\mathrm{n}=18) .100 \%(\mathrm{n}=21)$ had MDT management. Delivery: 43\%: vaginal delivery. 38\%: elective Caesarean section. 19\% emergency Caesarean section. Post-partum care: $100 \%$ babies had post exposure prophylaxis started within 4 hours. No babies contracted HIV. 100\% babies exclusively bottle-fed. $57 \%$ mothers given carbegoline.

Discussion/conclusion There was good compliance with guidelines. All women received HAART and the MTCT rate was $0 \%$. $43 \%$ of women had a vaginal birth. Inclusion of the importance of carbergoline in departmental training may improve compliance in this area. In a low prevalence centre a specialist HIV antenatal clinic cannot be justified. The centre has introduced bimonthly MDT meetings to discuss these cases and these results suggest that communication and standards of care are high.

\section{P209 OUTCOMES OF PARTNER NOTIFICATION (PN) FOR SEX PARTNERS OF PEOPLE WITH CHLAMYDIA, MANAGED VIA THE ONLINE CHLAMYDIA PATHWAY.}

\footnotetext{
${ }^{1,2}$ Jo Gibbs* ${ }^{*}$ 'Pam Sonnenberg, ${ }^{3}$ Laura Tickle, ${ }^{1}$ Lorna Sutcliffe, ${ }^{4}$ Voula Gkatzidou, ${ }^{4}$ Kate Hone, ${ }^{2}$ Catherine Aicken, ${ }^{5} S$ Tariq Sadiq, ${ }^{1}$ Claudia Estcourt. ${ }^{1}$ Queen Mary University of London, London, UK; ${ }^{2}$ University College London, London, UK; ${ }^{3}$ Barts Sexual Health Centre, London, UK; ${ }^{4}$ Brunel University London, Uxbridge, UK; ${ }^{5}$ St George's, University of London, London, UK
}

\subsection{6/sextrans-2016-052718.257}

Background/introduction Within the $e \mathrm{STI}^{2}$ consortium, we conducted exploratory studies of an innovative Online Chlamydia Pathway (OCP: results service, automated clinical consultation, electronic prescription via community pharmacy, with telephone helpline support), which included optional online partner management.
Aim(s)/objectives To evaluate PN outcomes and sex partner uptake of online management.

Methods Participants: untreated GUM clinic attenders (two London services) and people tested through six London NCSP online postal testing areas: 21.07.14-13.3.15. The OCP offered index patients an SMS/email containing a unique code and link to forward to partners permitting them to access online care via OCP (patient referral). Outcomes were captured by OCP and index-reported at telephone follow-up (2 weeks post diagnosis)

Results Outcomes: 221 index patients consented to the study and $172(78 \%)$ were followed up by telephone (median age 23, $62 \%$ female). These 172 index patients reported 371 partners; $317 / 371(85 \%)$ were contactable and $256 / 317$ (81\%) of these were notified. Index patients reported 120/317 (38\%) as treated. Online outcomes: 154 index patients reached PN stage of OCP (some had already been routed to clinic). 94/154 (61\%) requested online partner access. They reported 280 partners: 28 went online; 19 received treatment at their chosen pharmacy; and 4 were treated elsewhere.

Discussion/conclusion 38\% of partners treated compares favourably with outcomes for routine PN within similar studies. Online management of sex partners through patient referral is feasible but uptake was low and most successful PN was achieved offline. Pathway optimisation could include anonymised sex-partner PN messaging and provision of partner STI self-sampling kits.

\section{P210 THE PREVALENCE OF CHLAMYDIA IN PREGNANT WOMEN COMPARED WITH NON-PREGNANT WOMEN IN A BUSY SEXUAL HEALTH CLINIC IN THE UK: MAKING THE CASE FOR SYSTEMATIC CHLAMYDIA SCREENING IN PREGNANCY?}

Cornelia Junghans*, Katherine Warren, Dale Coley, Eleanor Draeger. Lewisham and Greenwich NHS Trust, London, UK

\subsection{6/sextrans-2016-052718.258}

Background Chlamydia trachomatis (CT) is the commonest STI in the UK with high prevalence in pregnancy. CT testing is not

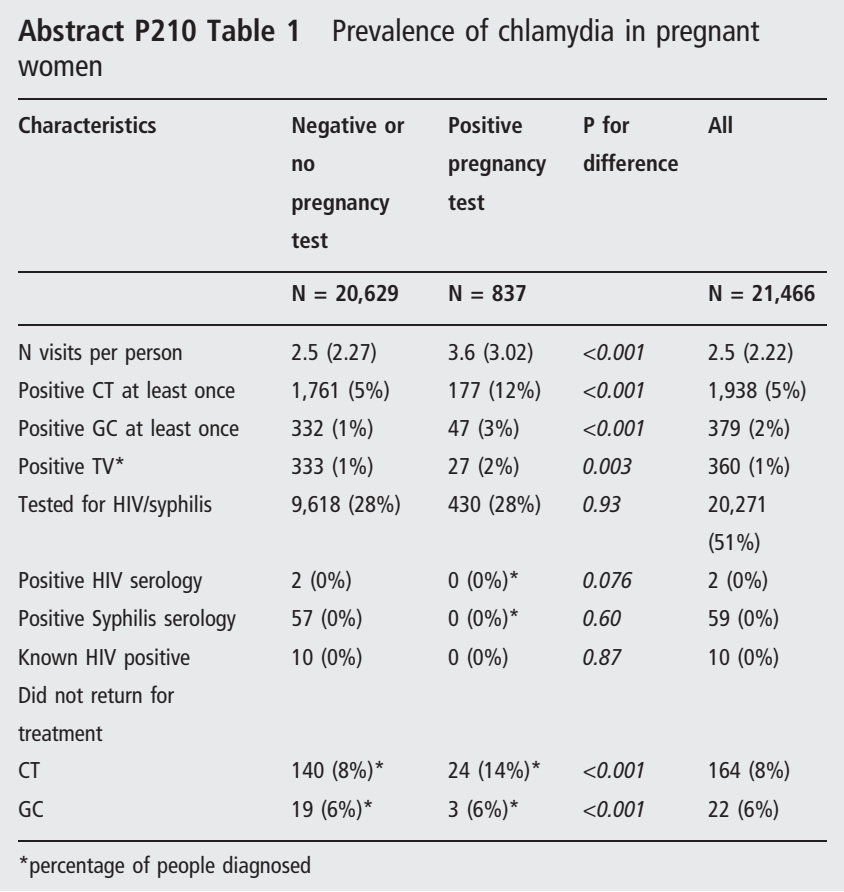

